\title{
Precursive Time, the Hidden Variable
}

\author{
Renè Burri \\ Free Academy of Modern Physics, Lugano, Switzerland \\ Email: r.burri@ietclab.org
}

How to cite this paper: Burri, R. (2020) Precursive Time, the Hidden Variable. Journal of Applied Mathematics and Physics, 8, 1135-1154.

https://doi.org/10.4236/jamp.2020.86086

Received: March 16, 2020

Accepted: June 19, 2020

Published: June 22, 2020

Copyright (c) 2020 by author(s) and Scientific Research Publishing Inc. This work is licensed under the Creative Commons Attribution International License (CC BY 4.0).

http://creativecommons.org/licenses/by/4.0/

\begin{abstract}
In this paper, a new complex variable defined as "precursive time" able to correlate general relativity (GR) and quantum field theory (QFT) in a single principle was characterized. The thesis was elaborated according to a hypothesis coherent with the "Einstein's General Theory of Relativity", making use of a new mathematical-topological variety called "time-space" developed on the properties of the hypersphere and explained mathematically through the quaternion of Hurwitz-Lipschitz algebra. In this publication we pay attention to the interaction between the weak nuclear force theory (EWT) and the nuclear mass of the Standard Model.
\end{abstract}

\section{Keywords}

Time Curvature, Precursive Time, Hidden Variable, Timespace Manifold, Chronotope, Quantum Compensation Spacetime

\section{Introduction}

In the previously published study (HDTSS 2016) ${ }^{1}$, we have exposed the thesis of a plausible "time curvature" induced by spacetime modifications. The hypothesis is coherent with GR which assumes that time curves jointly with space this is by definition.

The plausibility of the "time curvature" finds its theoretical hypothesis in the different classical representations of the "curvature" of spacetime, differently interpreted as a dynamic of a "twisting" process of spacetime.

From aconceptual point of view, the twisting dynamic implies a contraction effect of spacetime; the theoretical difference between "curvature" and "twist (torsion)" is decisive in the analysis of the topological variety of the system. The representation of space-time curvature is specifically described through the ten${ }^{1}$ Burri, R. (2016) Vacuum-Matter Interaction through Hyper-Dimensional Time-Space Shifting. Journal of High Energy Physics, Gravitation and Cosmology, 2, 432-446. http://dx.doi.org/10.4236/jhepgc.2016.23037. 
sor equations as solved by the Einstein Field Equation (EFE), in other words, the contracting space-time places the time variable inside a concept equation:

$<$ minor space $\Leftrightarrow$ minor time $\Rightarrow$ less space $\rightarrow$ less time $>$

\section{Theoretical Synopsis}

Theoretically, the time curvature can be described as: the projection of instantaneous time onto an imaginary abscissa shifted in advance towards the future.

However, more concretely we can see it as a "time discrepancy" $(\Delta \tau)$ that originates between the event's instantaneous time and its imaginary projection in spacetime. These results in a coherent representation of curved spacetime referred to a generic mass but specified by Planck constants. The topological characteristic of the representation of a time discrepancy or a spacetime-induced time warp was developed by placing as a reference theoretical concept: Minkowski4 spacetime ${ }^{2}$ [1]-[7]. The Minkowski4 manifold highlights the position in Euclidean space of an event recorded in an instant "chronotope" time, however it does not make possible a connection to an imaginary temporal base. In order to operate on the imaginary time variable, the study has developed a specific topological manifold applied to the properties of the Hypersphere 3S Sphere [8]-[12] manifold, which made it possible to represent a complex imaginary time related to the frame of reference of instantaneous time. The studied manifold variety, defined as "time-space", characterizes a pseudo-connection of correlative order between the time referred to the Minkowski space, and the projection on an imaginary timeframe of reference.

Time-space manifold resolves the correlation between <Minkowski4 space> and the projection of imaginary timeframe of reference elaborated based on the higher dimensions' geometry of the 3-s Sphere hypersphere, running an entanglement-type correlation. The time-space variety allows us to specify the imaginary part of the time axis leaving the space coordinates invariant, as shown in Figure 1 For the sake of simplicity, the graphical representation of the hypersphere is dashed at a semi-circle.

- $P_{k}$ : chronotope event

- $t_{0}$ : time event

- C: pseudo connection

- $e_{0}$ : Imaginary time $\left(e_{0} \equiv t_{0}\right)$ The reference point $\left(t_{0}\right)$ of chronotope time component expressed by $M 4$ space is correlated and in phase with its imaginary time frame of reference component $\left(e_{0}\right)$ when in a theoretical flat space-time condition.

${ }^{2}$ Minkowski4 spacetime provides a logical representation of our Universe considering that there is no event without a reference to a specific instant in time. Any event of ponderable nature is necessarily characterized by a precise and unique set of "space + time" coordinates, called for this reason "chronotope". The representation of the chronotope on a hyperplane or four-dimensional space establishes the uniqueness of that precise spacetime event. The chronotropic coordinates of an event establish the inviolability defined by the "Pauli exclusion principle". In fact, in the same place and at the same time there can be just one fermion. The chronotope representation on 4-dimensional space was the synthesis of the study developed by Hermann Minkowski to provide a coherent geometry to relativistic theories. 


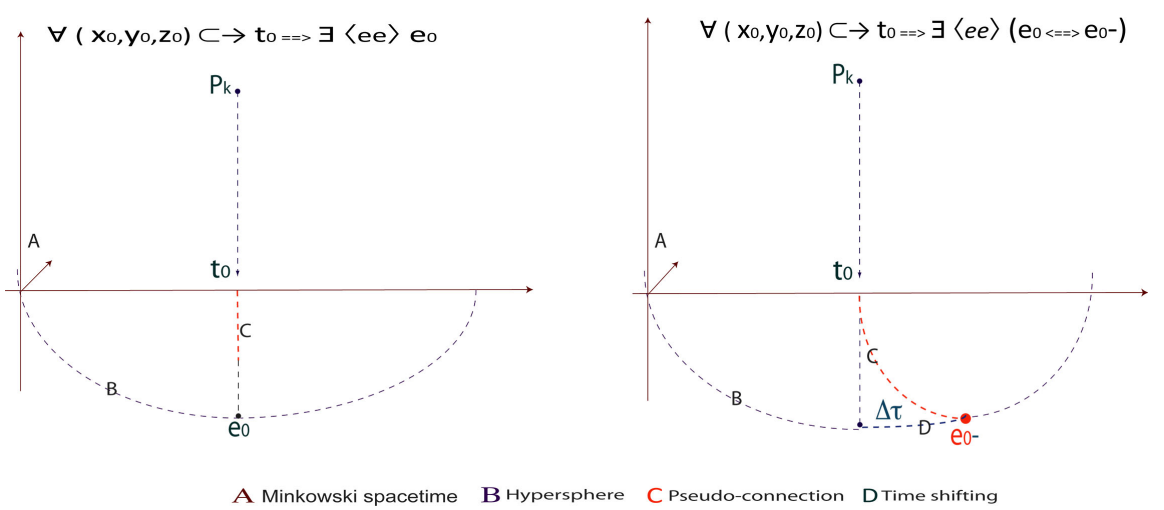

Figure 1. Correlation time event $\rightarrow$ projection Imaginary time in flat space $\left(e_{0}\right)$ and curved spacetime $\left(e_{0^{-}}\right)$In the case of flat space, $\left(e_{0} \equiv t_{0}\right)$ the two components (real and imaginary) coincide.

- B: hypersphere 3-s Sphere ( $\left\langle e_{0}\right\rangle$ is centre of gravity)

- D. discrepancy time - time-space curvature $(\Delta q t)$

- $e_{0^{-}}$: precursive time $\left(t_{0} \Leftrightarrow e_{0^{-}}\right)$Chronotope event " $P_{k}$ " with time component $\left(t_{0}\right)$, establishes a new correlation $\left(e_{0^{-}}\right)$becomes the new point of hypersphere centre of gravity.

\section{TimeSpace Manifold}

The use of the term "timespace", at variance from spacetime, makes sense because in the thesis we take the parameter time as the independent function and space as the dependent function. In fact, the term "chronotope" [13] [14] is composed, in the order, by: chronos (time) + topos (space) $\equiv$ time space.

The "time-space manifold" can be understood as an entanglement-type connection with symbol "<ee>" between |Minkowski4 space| and <3-s Sphere hyperspace $>$, mathematically it is expressed according to the quaternion algebra.

$a(x) ; b(y) ; c(z)$, and time component: $\left|d\left(t_{0} \rightarrow e_{0}\right)\right|$; pure quaternion part:

$$
\begin{gathered}
\left(x_{i}, y_{j}, z_{k}\right) \\
\forall\left(x_{0}, y_{0}, z_{0}\right) \in \rightarrow t_{0} \Rightarrow\left\langle e_{0}\right\rangle\left(t_{0} \leftrightarrows e_{0}\right) \in\left(x_{i}, y_{j}, z_{k}\right) \subset\left(e_{0} \Leftrightarrow e_{0-}\right) \\
\forall\left|P_{k}\right| \in \mathbb{R}\left(x_{0}, y_{0}, z_{0}\right) ; \mathbb{C}\left(x_{i}, y_{j}, z_{k}, e_{0-}\right) \\
\mathbb{R}\left(x_{0}, y_{0}, z_{0}\right) ; \mathbb{C}\left(x_{i}, y_{j}, z_{k}, e_{0-}\right)
\end{gathered}
$$

$\left(x_{i}, y_{j} z_{k}\right) \equiv \sigma$, hyperspace coordinate o ( $\sigma$ sigma-point)

The expression can be described according by Hurwitz-Lipschitz [15] [16], since the components are unitary quantities:

$$
\begin{gathered}
{\left[\begin{array}{cccc}
-e_{0}+t_{0} & 0 & 0 & 0 \\
0 & x_{0}-x_{i} & 0 & 0 \\
0 & 0 & y_{0}-y_{j} & 0 \\
0 & 0 & 0 & z_{0}-z_{k}
\end{array}\right]} \\
{\left[\left\{-e_{0}+t_{0}, 0,0,0\right\},\left\{0, x_{0}-x_{i}, 0,0\right\},\left\{0,0, y_{0}-y_{j}, 0\right\},\left\{0,0,0, z_{0}-z_{k}\right\}\right]}
\end{gathered}
$$




$$
\left[\left\{-e_{0}+t_{0}, x_{0}-x_{i}, y_{0}-y_{j}, z_{0}-z_{k}\right\}\right]
$$

The "precursive time" $\left(e_{0-}\right)$ is the variable that quantifies the "time curvature" effect that is generated between the instant time of the event and the projection on spacetime.

$$
\left(t_{0} \preceq e_{0} \Leftrightarrow e_{0-}\right)
$$

The concept of time curvature is expressed by the "time-space" complex manifold (Figure 2).

The timespace manifold layout is represented by two hemispheres which respectively indicate: (red) the mass relative to the event and (blue) the hyper-dimensional component temporally shifted with respect to the inertial frame reference of the chronotope.

- $P_{k}$ : chronotope (event)

- $t_{0}:$ real time event

- $e_{0}$ : Imaginary projection event time (Hypersphere centre of gravity)

$-e_{0-}$ : precursive time event

- M4: Minkowski4 space

- 3-Sph; hypersphere 3-s Sphere

The spacetime variation induced by a generic mass (red hemisphere) is quantifiable by its time curvature that is generated between the instantaneous real time $\left(t_{0}\right)$ and the correlated complex imaginary time: $\left(e_{0} \rightarrow e_{0_{-}}\right)$.

The manifold "timespace" allows us to depict the concept of precursive time $\left(\Delta \tau, e_{0-}\right)$. The connection points are respectively: the centre of gravity $\left(P_{k}\right)$ and the hypersphere centre of gravity (3-Sph).

\subsection{Time Curvature Conceptual Meaning}

The proportionality correlation that is determined between the inertial mass referred to the event and the time curvature that it generates on spacetime allows us to describe the spacetime variations by operating via quantum field theory (QFT).The invariance to the reference system as defined by the Lorentz transformations is preserved by the event's frame of reference (Figure 3 ).

Calculations of time curvature $(\Delta \tau)$ and precursive time $\left(e_{0_{-}}\right)$as referred to a generic inertial mass.

The theses and concepts from which the main equations were obtained were described in the previous HDTSS publication, [17]; in this study we make some improvements in the calculation and further analyze and evaluate the procedure. We will examine a fundamental correlation that occurs between: < nuclear mass weak nuclear force-orbital levels>.

\subsection{Time Curvature Equation}

The time-curvature $(\Delta q t)$ is calculated in Planck time.

The Equation (1) allows us to determine time-space curvatures $(\Delta \tau)$ of a generic inertial mass: 


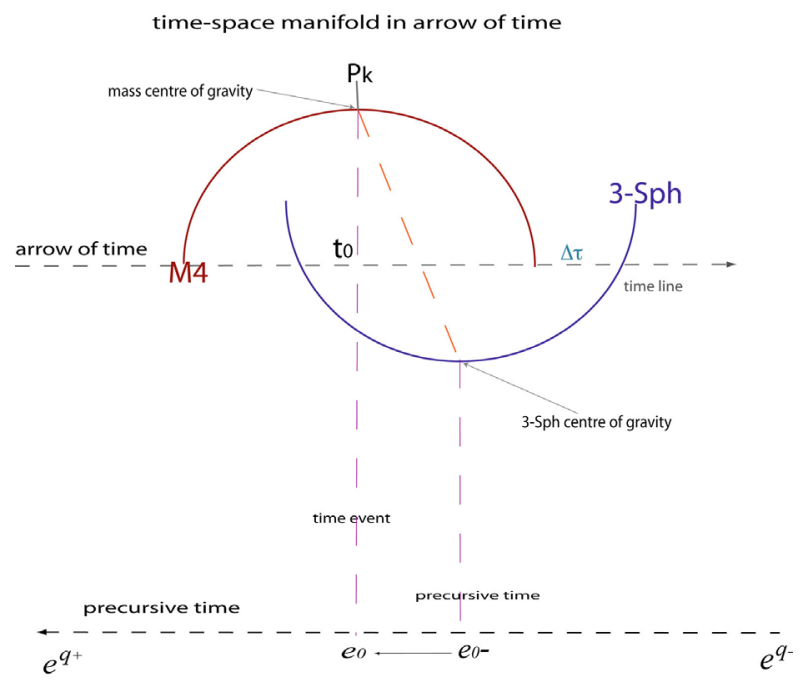

Figure 2. Timespace manifold depiction: The connection points are respectively: the centre of gravity the mass event and the centre of gravity the hypersphere 3 -Sph (red hemisphere). The "precursive" time discrepancy $(\Delta \tau)$ specifies exactly he inertial mass value (blue hemisphere). Notice that the instantaneous time imaginary projection undergoes a shift as a function of the inertial mass.

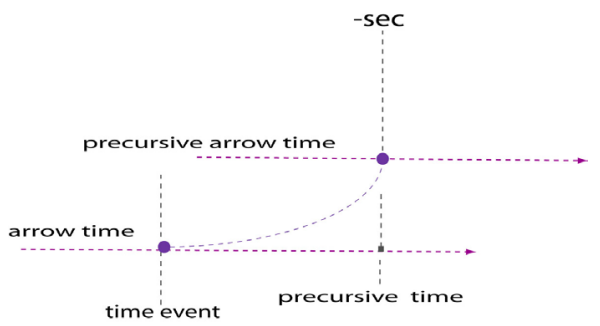

Figure 3. In this figure, we distinguish the event on the arrow of time as in the classic scalar representation and on the imaginary abscissa the related "conjugate time" (-sec.).

$$
M\left(P_{k}\right)=\frac{h}{t_{p} c^{2}} \rightarrow t\left(P_{k}\right)=\frac{h}{M\left(P_{k}\right) c^{2}} \rightarrow \frac{1}{\sqrt{\frac{h}{M\left(P_{k}\right) c^{2}}}}(s)^{-1} \Delta \tau(\text { joules } / \mathrm{sec})
$$

- $t_{p}=\sqrt{\frac{\hbar G}{c^{5}}}$ Planck time

- $M\left(P_{k}\right)=$ Planck mass event

$-c^{2}=$ Speed of Light Squared

From the Equation (1) we get the value of the precursive time $\left(e_{0-}\right)$ (-seconds) referred to the instant time inertial frame of the reference event.

\subsection{Precursive Time Meaning}

$$
\tau_{v}=\frac{1}{\sqrt{\frac{h}{m_{p} c^{2}}}} \text { joules } / \text { sec }
$$

As Figure 4 shows, Equation (1) determines the amount of deflected time in- 
duced by the time curvature of the inertial mass of the event; we can distinguish:

- $(\Delta \tau)$; time curvature: $(\Delta \tau)$ indicates the deflection effect of the real time coordinate induced by the time curvature.

- $\left(\tau_{v}\right)$; precursive time: the precursive time is specifically the amount of time deflected by the time curvature. We remember that the time deflection effect is dictated by the equation:

$<$ minor space $\Leftrightarrow$ minor time $>$ so $<$ less space $\rightarrow$ less time $>$. From a relativistic point of view, the dynamics (torsion $\rightarrow$ contraction) of spacetime simultaneously determines a deflation effect of space (less space) together with a deflection of time (less time):

|time deflection $\rightarrow$ space deflation $\mid \Rightarrow$ spacetime variation

Consequently, the time curvature becomes the primary cause of the spacetimecurvature according general theory of relativity (Figure 5).

It is important to note that $\left(\tau_{v}\right)$ expressed in Planck time (joule/sec) coincides with the amount of energy of the inertial mass:

$$
\mid \operatorname{energy}\left(\tau_{v}\right) \equiv \text { inertial mass } \mid \Rightarrow(\Delta \tau) \equiv M i
$$

The equation states that the inertial mass is induced by the time curvature; more specifically we can state that the inertial mass is exactly the value the time curvature.

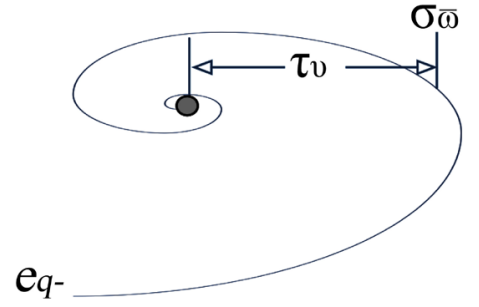

Figure 4. Equation (1) specifies the time curvature generated by the event in spacetime. The dynamic is centripetal, because it precursive, whereby involves from the future $\left(e_{0-}\right)$ toward the time event $\left(t_{0}\right)$.

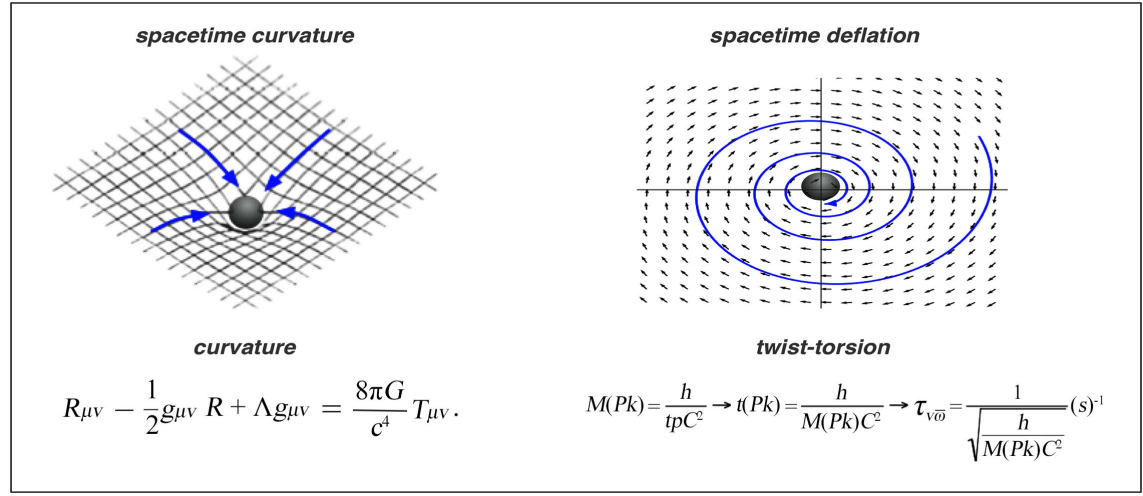

Figure 5. Depiction as "spacetime curvature" and "spacetime deflation". The depiction of spacetime in twist dynamics allows us to act on hyper-dimensional coordinate working with quantum correlations. 
Two other fundamental parameters are acquired from Equation (1):

1) $(-s)$ time deflection in second (Planck time in precursive second).

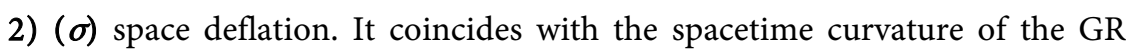
(Figure 5).

Equation (1) shows that spacetime variations can be interpreted as classically described by GR through quantum physics.

Equation (1) also confirms that:

$<$ For each event with [mass $>0$ ] there is an imaginary time component proportional to the value of the inertial mass $>$.

Equation (2) extends the concept of complex variable mass in which the inertial frame of reference is a function of the time-space curvature $(\Delta \tau)$, the equality $(m i \equiv \Delta \tau)$ indicates that:

Theinertial mass in the absence of interacting gravitational forces is a physical quantity determined by the time curvature.

Note:

Conceptually, the hypothesis of time curvature deals with two important inexplicable issues that cannot be demonstrated through the classic depiction of space-curved GR:

1) It unifies general relativity and quantum field theory.

2) It unifies inertial and gravitational mass phenomena.

\subsection{Time Space Quadrant as a Hyper-Dimensional Manifold}

The representation in TimeSpace quadrant allows us to get a photograph of the event in instant time, as a "snapshot" of the imaginary timespace curvature $\left(\sigma_{\bar{\sigma}}\right)$. The coordinates $\left(x_{i}, y_{j}, z_{k}\right)$ can be resolved as "hyperdimensional" since the complementary reference base $\left(e_{0} \rightarrow e_{0-}\right)$ is placed on an abscissa dimensionally shifted with respect to the event (instantaneous time) (Figure 6).

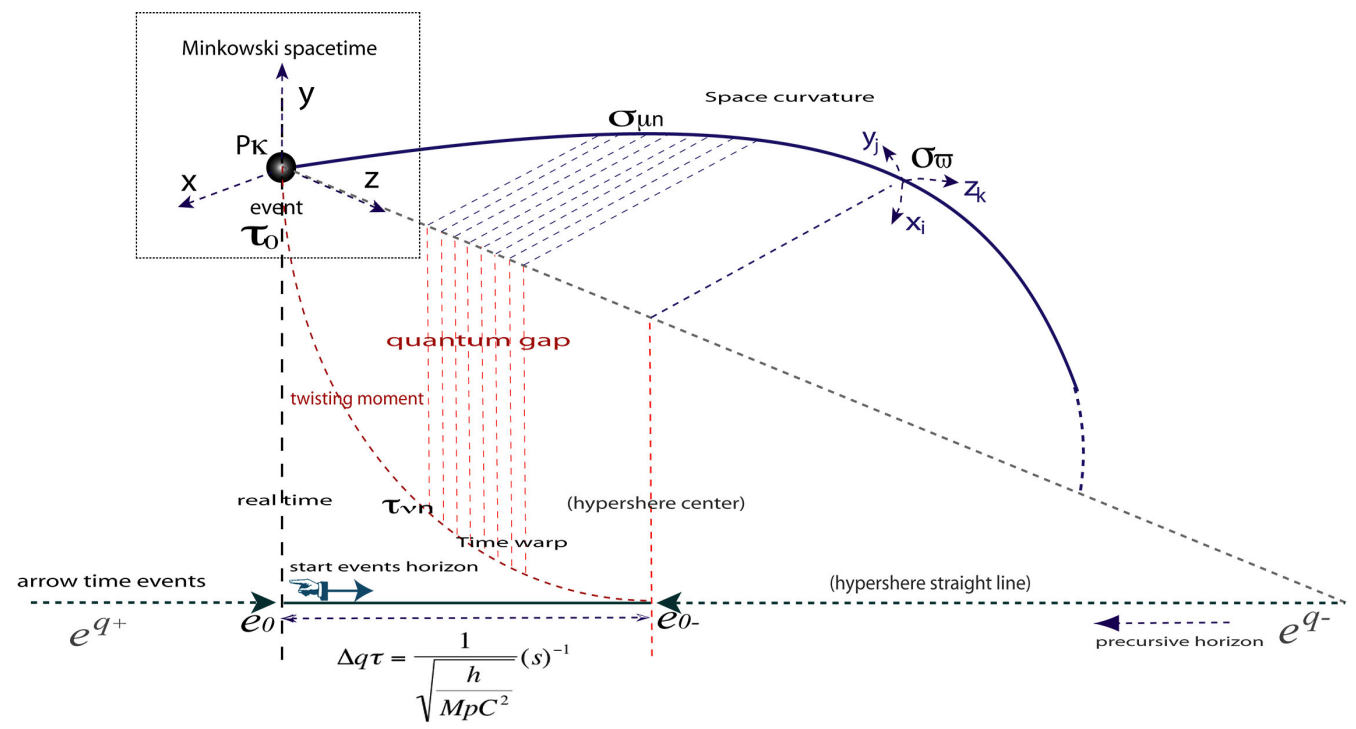

Figure 6. Representation in quadrant depiction; this allows us to have a comprehensive picture of the coordinates as referred to the event. 
- Coordinates $\left(x_{i}, y_{j}, z_{k}\right)$ define the "curvature" of the deflated space.

- Sigma point $\left(\sigma_{\varpi}\right)$ which indicates the point of deflated edge space (is the concrete physical space even if calculated with imaginary coordinates).

- $\left(e_{0-}\right)$ is a deflection Imaginary time coordinate assuming the local observables reference frame, induced by the inertial mass $\left(P_{k}\right)$ on time space (time curvature) $\left(e_{0-}\right)$ is positioned in advance (precursive) with respect to $\left(t_{0}\right)$, because $<$ less space $\rightarrow$ less time $>$.

\subsection{Time Curvarture Coordinates}

From Timespace manifold:

$$
\begin{gathered}
\forall\left|P_{k}\right| \in \mathbb{R}\left(x_{0}, y_{0}, z_{0}\right)\left(t_{0}\right) \subseteq \mathbb{C}\left(x_{i}, y_{j}, z_{k}, e_{0-}\right) \\
M 4 \mathbb{R}\left(x_{0}, y_{0}, z_{0}, t_{0}\right) ; 3 s \mathbb{C}\left(x_{i}, y_{j}, z_{k}, e_{0-}\right) ; \\
P_{k}\left(t_{0}\right)\left(x_{0}, y_{0}, z_{0}\right) ; P_{k}^{\prime}\left(e_{0-}\right)\left(x_{i}, y_{j}, z_{k}\right) ;
\end{gathered}
$$

Summing up, each event is characterized by "seven" coordinates:

$$
P_{k}\left(t_{0}\right)\left(x_{0}, y_{0}, z_{0}\right) ; P_{k}^{\prime}\left(e_{0-}\right)\left(\sigma_{\sigma}\right)
$$

real coordinates referring to the chronotropic position of the M4 event:

$$
P_{k}: \mathbb{R}\left(x_{0}, y_{0}, z_{0}\right)\left(t_{0}\right)
$$

and four 3S hyperdimensional coordinates:

$$
\sigma_{\sigma}: \mathbb{C}\left(x_{i}, y_{j}, z_{k}\right)\left(e_{0-}\right)
$$

where, $\left(t_{0}\right)$ is the event time of inertial frame of reference.

\subsection{Calculation of Inertial Mass as a Function of Spacetime}

From the analyses carried out in this study, we can assume that matter and spacetime are in fact a single entity consisting of two joint parts, real partand spacetime the complex hyperdimensional joint part, the interaction <inertial mass $\Leftrightarrow$ spacetime $>$ assumes the concept of a bivalent set. Ref. timespace manifold (Figure 2).

From Equation (1), we can infer correlation (3) that introduces a concept of mass expressed in Planck parameters, directly related to the spacetime interaction properties.

It is a new expression of mass calculation as a function of the precursive time, that is, time-space curvatures, a new concept of mass that we distinguish with the name "mass-matter" $(\psi)$.

Equation (3): calculation of inertial mass according to the precursive time equation:

$$
\tau_{v}=\frac{1}{\sqrt{\frac{h}{m_{p} c^{2}}}} \rightarrow \psi=\frac{m_{p} \tau_{v}}{\Gamma_{p} K} \pi^{F}
$$

- $\quad \psi$ : mass-matter (inertial mass as function oftime curvature) 
- $\boldsymbol{m}_{p}$ : chronotope mass $\left(P_{k}\right)$ in Planckmass

- $\tau_{v}$ : precursive time

- $\Gamma_{p}$ : quantum specific Heat capacity.

- K: Boltzmann Constant

- $\pi^{F}$ : quantum compensation spacetime, (F) Fibonacci coefficient [18] [19] [20] [21].

The equation of the calculation of the inertial mass as a function of the timespace curvature implies a direct correlation to Quantum Heat capacity $\left(\Gamma_{p}\right)$ :

\section{Q. Heat capacity:}

$$
\Gamma_{p}=\frac{\tau_{v}}{K} C^{2}
$$

The equation expressed with $\left(\Gamma_{p}\right)$ is calculated as Planck temperature referred to $\left(\tau_{v}\right)$.

Equation (3) highlights that: $\psi f\left(\Gamma_{p} \rightarrow \tau_{v}\right)$

this leads to asserting that $\left(\Gamma_{p}\right)$ is a state property of matter.

From the calculation of Q.Heat capacity it is pointed out that is a characteristic of the mass as a function of the time curvature; in fact the correlation provides us with a reading key to the interpretation of the rebus of absolute zero: $\left(\psi \rightarrow \Gamma_{p}\right)>0 \mathrm{~K}$

From the correlations expressed, we can say that: a generic mass $|m i>0|$ has a heat state of Q. Heat capacity determined by its time curvature. This assumption implies that it cannot degrade below the Absolute Zero (0 K).

\subsection{Quantum Compensation Spacetime $\left(\pi^{F}\right)$}

\section{(F) Fibonacci coefficient}

Let's highlight the remarkable coefficient $\left(\pi^{*}\right)$ obtained from theoretical processing of spacetime twist dynamics: quantum compensation spacetime (q.c.s.). This coefficient allows us to obtain aprecise measure of the value of the inertial mass as a function of the precursive time. The representation of spacetime calculated as a function of the Fibonacci exponential faithfully describes the variation of spacetime in twist (torsion) dynamics with an accuracy of the time curvatures of $\left(10^{-15}\right)$, as shown below in Figure 8 and Figure 9 with regard to nuclear masses. The constant of "quantum compensation spacetime", describes the development layout of the twist-contraction dynamics of spacetime (Figure 7).

Given the coordinates: $\left(y i_{(e 0)} ; x i_{(e 0)}\right)$ the graphic elaboration model draws a centripetal cycloid where the space-time (eigenvectors) contracts towards the centre of gravity of the chronotope. The four segments $(F)$ represent equipotential space-time areas with a different frame of reference.

It is important to underline that the different areas or equipotential regions $\left(F_{1}, F_{2}, F_{3}, F_{4}\right)$ are also iso-temporal, this being in accordance with Equations (1) and (2). The summation of Fibonacci's hyperdimensional regions: complex time + complex space, is equivalent to the value of the inertial mass as referred to the chronotope: $\left(F_{n}\right): \tau_{v_{n 1}}+\tau_{v_{n 2}}+\tau_{v_{n 3}}+\tau_{v_{n 4}}=\psi$ 

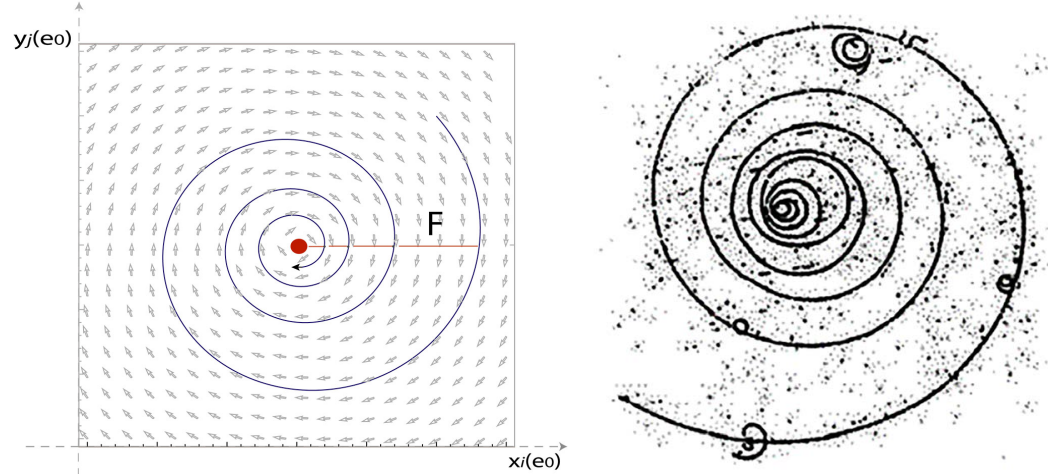

Figure 7. Left image: computer processing of space-time torsion contraction, Coordinates (Yj and $\mathrm{Xi}$ ) draw a centripetal cycloid with a Fibonacci progression $\left(\pi^{F}\right)$ (Spiral sinks complex eigenvalues graphics processing). Right image: comparison with a spiral generated by an electron in a bubble chamber -Photo: Harvard project, Elementary Particles [22].

The hypothesis under study seems to draw an important meaning as a physical consequence, that is: mass and spacetime are not two different interacting entities as we would interpret classically, but we can state that:

$<$ inertial mass and spacetime are constituents of a single entity in which precursive time is the mediator $>$.

\subsection{Energy Equation}

The energy equation $|\psi|$ function of time curvature, becomes a concept of mass peculiarly related to spacetime interaction. As a result of this, the equation: $\left(E=m c^{2}\right)$, can be reexamined in:

$$
E=\psi c^{3}
$$

Equation (5) which confirms the thesis, in fact it can take over from Einstein's equation.

$$
E=m c^{2} \equiv \psi c^{3}
$$

From the exposed correlations [matter $\rightarrow$ precursive time $\rightarrow$ energy], we can support the principle stating that: [matter $\Leftrightarrow$ spacetime] uniquely phenomena.

\section{Unified General Relativity $\Leftrightarrow$ Quantum Field Theory}

In this unpublished second part of the publication we submit the working hypothesis to the test of coherence applied to the nuclear masses. The theses that are here developed involve an atypical research approach, as general relativity (GR) and quantum field theory (QFT):

\subsection{Correlation: Timespace Curvature $\Leftrightarrow$ Nuclearenergy}

Figure 8 shows an example of application of Equations (1) (3)-(5) to the nuclear masses of the following atomic elements.

Figure 8 confirms that the correlations are consistent, in fact the value of the energy calculated in $\mathrm{GeV}$ is correct. 
The value of the energy GeV confirms: [ $E=m c^{2} \equiv \psi c^{3}$ ]

- Column $(\Delta \tau) \mathrm{J} / \mathrm{sec}$ indicates the precursive time (time curvature) calculated from Equation (1).

- Column ( $\Psi)$ indicates "mass-matter" calculated from Equation (3).

- The energy calculation $(\mathrm{GeV})$ is obtained from Equation (5).

Figure 8 confirms the thesis that:

1) We can specify spacetime in quantum physics.

2) Spacetime can also be calculated for atomic nuclei.

In Figure 9, it is possible to quantify the spacetime variation even for nuclear masses. We apply Equations (1) (3)-(5) to the nuclear masses of the following atomic elements:

\subsection{Correlation: Timespace Curvature $\Leftrightarrow$ Electron Energy Levels}

The column (picometers) is calculated:

- $(\Delta q t): \tau_{v} \Rightarrow P t c^{2}=$ orbital radius

- nuclear mass $\psi c^{3}=\mathrm{GeV}$

Figure 9 correlates time curvatures $(\Delta \tau)$ and the distance $\left(\sigma_{\sigma}\right)$ in picometers.

We can note that time curvatures generated by the nucleus' mass determines the distance of the electronic orbitals' levels $\left(\sigma_{\sigma}\right)$.

\begin{tabular}{|c|c|c|c|c|c|c|c|}
\hline elements & un.atomic mass (u) & Mass $(\mathrm{Kg})$ & planck mass & $\Delta \mathrm{T}$ (joules/sec) & $\psi$ mass-matter & $\psi \mathrm{C} 3$ & GeV \\
\hline Hydrogen & $1,0079400 E+00$ & $1,6726200 \mathrm{E}-27$ & $7,6900000 \mathrm{E}-20$ & $3,2289743 E+15$ & $5,57933 \mathrm{E}-36$ & $1,50329 \mathrm{E}-10$ & 0,9382824 \\
\hline $\mathrm{Li}$ & $6,9400000 \mathrm{E}+00$ & $1,1500000 \mathrm{E}-26$ & $5,2950000 \mathrm{E}-19$ & $8,4729453 E+15$ & $3,8414 \mathrm{E}-35$ & $1,03503 \mathrm{E}-09$ & 6,460129 \\
\hline $\mathrm{Na}$ & $2,2989769 \mathrm{E}+01$ & $3,8175409 \mathrm{E}-26$ & $1,7540000 \mathrm{E}-18$ & $1,5421131 \mathrm{E}+16$ & $1,27249 \mathrm{E}-34$ & $3,42859 \mathrm{E}-09$ & 21,39956 \\
\hline $\mathrm{K}$ & $3,9098300 \mathrm{E}+01$ & $6,4924300 \mathrm{E}-26$ & $2,9830000 \mathrm{E}-18$ & $2,0110740 E+16$ & $2,1641 \mathrm{E}-34$ & $5,83094 \mathrm{E}-09$ & 36,39386 \\
\hline $\mathrm{Rb}$ & $8,5467800 \mathrm{E}+01$ & $1,4192000 \mathrm{E}-25$ & $6,5210000 \mathrm{E}-18$ & $2,9734355 E+16$ & $4,73083 \mathrm{E}-34$ & $1,27468 \mathrm{E}-08$ & 79,55901 \\
\hline Cs & $1,3290545 E+02$ & $2,2069470 \mathrm{E}-25$ & $1,0140000 \mathrm{E}-17$ & $3,7078350 E+16$ & $7,35633 \mathrm{E}-34$ & $1,98209 \mathrm{E}-08$ & 123,7124 \\
\hline $\mathrm{Fr}$ & $2,2300000 \mathrm{E}+02$ & $3,7030000 \mathrm{E}-25$ & $1,7010000 \mathrm{E}-17$ & $4,8023474 \mathrm{E}+16$ & $1,23404 \mathrm{E}-33$ & $3,32499 \mathrm{E}-08$ & 207,5293 \\
\hline
\end{tabular}

Figure 8. This figure shows how the time curvatures equation can determine the energy value of the relevant respective nuclear. The calculated values are correct and confirm our thesis.

\begin{tabular}{|c|c|c|c|c|c|}
\hline elements & $\Delta$ T (joules $/ \mathrm{sec})$ & (PlanckTime) & Pt m*sec & $(\sigma \omega)$ Picometers & GeV \\
\hline \hline Hydrogen & $3,22897 \mathrm{E}+15$ & $1,741 \mathrm{E}-28$ & $1,56473 \mathrm{E}-11$ & 15,64733 & 0,9382824 \\
\hline $\mathrm{Li}$ & $8,47295 \mathrm{E}+15$ & $4,568 \mathrm{E}-28$ & $4,10551 \mathrm{E}-11$ & 41,05513656 & 6,460129 \\
\hline $\mathrm{Na}$ & $1,54211 \mathrm{E}+16$ & $8,314 \mathrm{E}-28$ & $7,47225 \mathrm{E}-11$ & 74,72250556 & 21,39956 \\
\hline $\mathrm{K}$ & $2,01107 \mathrm{E}+16$ & $1,084 \mathrm{E}-27$ & $9,74251 \mathrm{E}-11$ & 97,42506138 & 36,39386 \\
\hline $\mathrm{Rb}$ & $2,97344 \mathrm{E}+16$ & $1,603 \mathrm{E}-27$ & $1,4407 \mathrm{E}-10$ & 144,0704552 & 79,55901 \\
\hline $\mathrm{Cs}$ & $3,70783 \mathrm{E}+16$ & $1,999 \mathrm{E}-27$ & $1,79661 \mathrm{E}-10$ & 179,6611602 & 123,7124 \\
\hline $\mathrm{Fr}$ & $4,80235 \mathrm{E}+16$ & $2,589 \mathrm{E}-27$ & $2,32688 \mathrm{E}-10$ & 232,6877158 & 207,5293 \\
\hline
\end{tabular}

Figure 9. This figure calculates the time curvature $(\Delta \tau)$ generated by the mass of the nucleus. The space gap in picometers is exactly the electron distance from the nucleus according to its energy level. 


\section{The Electromagnetic, Weak, and Strong Interactions}

\subsection{Correlation: Nuclear Mass $\Leftrightarrow$ Nuclear Electronic Orbital Levels}

In the following diagram we have highlighted the correlation ratio determined between the orbitals i.e. $\mathrm{Na}$-sodium $(2,8,1)$ and the respective distance from the nucleus. The energy contained in orbitals $(2,8.1)$ is directly related to the distance between the nucleus and the orbital radius.

- Values in red refer to the energies $((\Delta \tau) \mathrm{J} / \mathrm{sec})$ ) of the different orbital levels.

- Values in blue refer to the positions $(\mathrm{Pm})$ in picometers attributed to orbitals.

The orbital takes on an energy value as a function of the distance determined from the nucleus.

From the Equation (1) $\left(\tau_{v}\right)$ it is possible to calculate the levels of the orbitals and the number of electrons between orbitals according to the distance from the nucleus.

Figure 10 shows the position of the three sodium levels and the overall energy equal to the number of electrons on that level:

\subsection{Correlation: $\Delta \tau \rightarrow \Gamma_{p}$ Exothermic $\leftrightarrow$ Endothermic $Q$ Value}

The movement of electrons between orbital levels in emission or absorption form is indeed a variation of the orbital radius, the correlation is:

$$
\left|\tau_{v}\right| \rightarrow\left(\sigma_{\bar{\sigma}}\right) \rightarrow \psi c^{3}
$$

Each level is characterized by a space position coordinate $\left(\sigma_{\mu n}\right)$ (Figure 11) and a corresponding amount of energy as a function of precursive time $\left(\tau_{v}\right)$. The complexity of the system is due to the double correlation that specifies the coordinate set $(\mathbb{R}+\mathbb{C})$ for each Orbital level $(K, L, M, N)$ :

- $\Sigma K: \mathbb{C}\left(x_{k i}, y_{k j}, z_{k k}\right)\left(\tau_{v_{k}}\right) \Rightarrow \psi_{k} c^{3}$

- $\Sigma L: \mathbb{C}\left(x_{l i}, y_{l j}, z_{l k}\right)\left(\tau_{v_{l}}\right) \Rightarrow \psi_{l} c^{3}$

- $\quad \Sigma M: \mathbb{C}\left(x_{m i}, y_{m j}, z_{m k}\right)\left(\tau_{v_{m}}\right) \Rightarrow \psi_{m} c^{3}$

- $\Sigma N: \mathbb{C}\left(x_{n i}, y_{n j}, z_{n k}\right)\left(\tau_{v_{n}}\right) \Rightarrow \psi_{n} c^{3}$

Nucleus:

- chronotope nucleus position (nucleus coordinate): $P_{k}: \mathbb{R}\left(x_{0}, y_{0}, z_{0}\right)\left(t_{0}\right)$

- Nucleus energy: $(\Delta q \tau) c^{3}$

- Atom cross sections: $\sigma_{\sigma}: \mathbb{C}\left(x_{i}, y_{j}, z_{k}\right)\left(e_{0-}\right)$

- $\operatorname{orbital}(\mathrm{N})$ stretch dynamic: $\left(\frac{1}{n_{e}^{2}}=\tau_{N 1}-\tau_{N 2}\right)$

$>\frac{m e^{4}}{8 \varepsilon_{0}^{2} h^{2}} \tau_{N 2} \rightarrow \Delta \tau\left(-\Gamma_{p}\right) \rightarrow$ orbital contraction (Figure 12).

$>\frac{m e^{4}}{8 \varepsilon_{0}^{2} h^{2}} \tau_{N 1} \rightarrow \Delta \tau\left(+\Gamma_{p}\right) \rightarrow$ orbital stretch (Figure 12).

In summary, each orbital consists of a space position $\left(\sigma_{\mu n}\right)$ (hyperspace posi- 
tioning) and a time quantum level $\left(\tau_{v}\right)$ which determines its energy (Figure 11). The correlation is extremely significant, as it places the nucleus mass as the cause of the energies distributed over the orbital levels (Figure 12 general diagram).

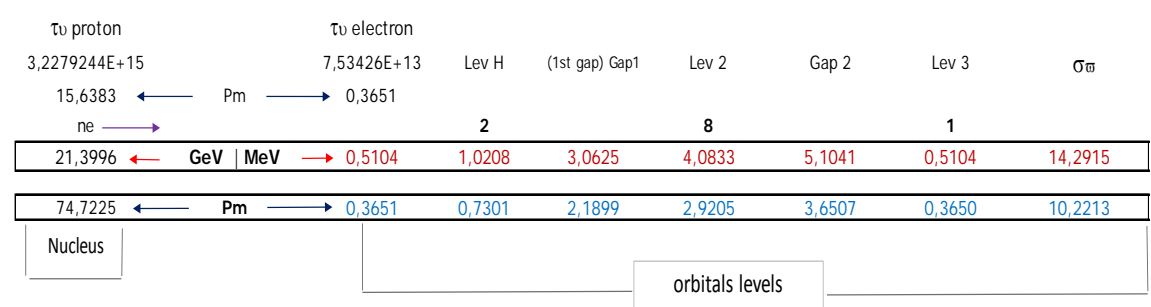

Figure 10. Orbital sodium levels obtained from a calculation model from Equation (1).

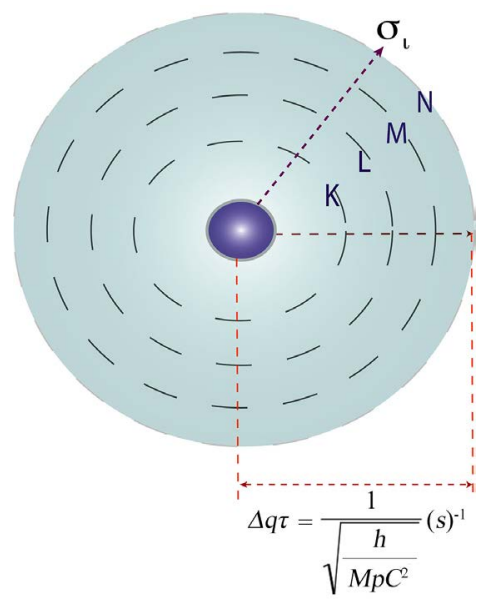

Figure 11. The correlation ratio between nucleus mass and orbital radius defines the electroweak force.

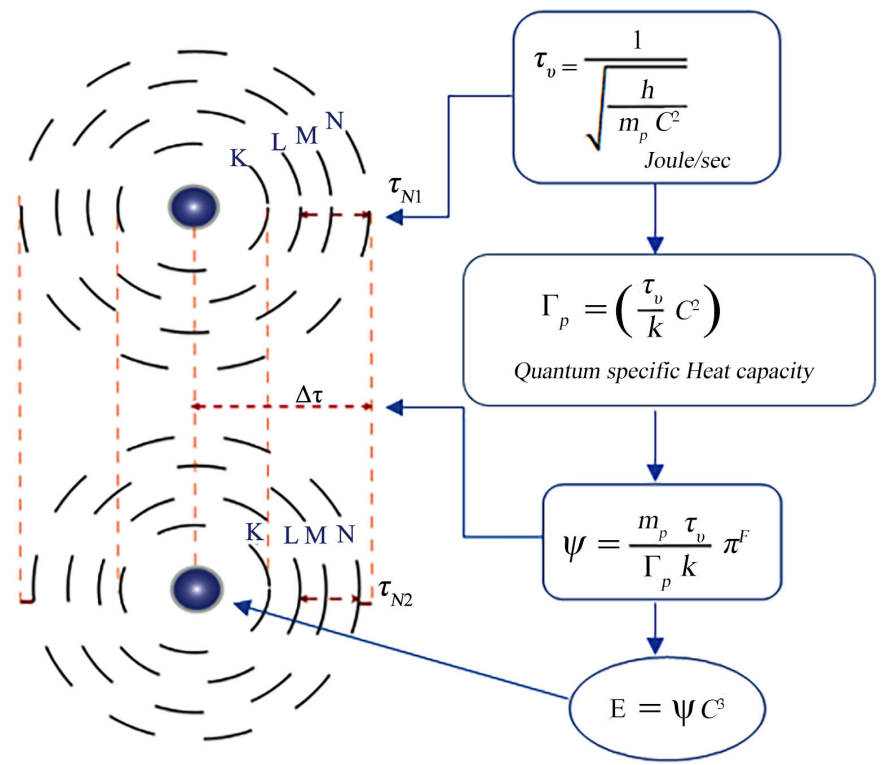

Figure 12. General diagram: The dynamic of the <exothermic $\Leftrightarrow$ endothermic $>$ reaction can be very similarly imagined to a (stretching dynamic). Slide show 2019 LANR/CF Colloquium at MIT, March 23-24, 2019. Cambridge MA 02139 (USA). 


\section{Theoretical Fallout}

\subsection{Extending the Standard Model in Hyper-Dimensional Mechanics}

As shown on the chart in Figure 11, the electrons are emitted from quantum time precursive orbital, with respect to the nucleus chronotypal position, (the frame of reference of the nucleus is delayed compared electronicorbitals), it follows that the electron does not have a specific position " $\mathrm{x}$ " and momentum "p", but is in a weak interactions' regime in the latency energy form of.

The deductive conclusions suggest an understanding of the structure of a four-dimensional atom much more complex than the one presented by the current "standard model".

The concepts presented so far are coherent with the standard model, Bohr model representation models hitherto analyzed, however the electroweak interaction dynamics defined by the new variable suggest a mathematical structure which goes under the name the Hopf fibration [23] [24] [25].

Hopf's fibrational dynamics configuration allows the standard model to be extended to hyperspace correlations between nucleus and electronic orbitals. Fibrational mechanics allow a consistent representation of the theory and allow to calculate the energies of the electronic orbitals (Figure 13 referred to Figure 10)

The fibration dynamics explain the reason why there is no conflict between the negative charge of the electron and the positive charge of the nucleus, the system to continue in balance because it is the nucleus itself the one generating the spacetime variations that give rise to the electron charge of the electro-weak interaction.

- fibration positioning $\left(s^{1} \rightarrow s^{3} \rightarrow s^{2}\right)$.

- energy levels $(\psi 1 \rightarrow \psi 3 \rightarrow \psi 2)$.

The proposed atom fibration depiction dynamics does not modify the energy levels ascertained by the standard model, it varies the rationality of the origin causality of the Electroweak interactions.

As regards Quantum Chromodynamics (QCD) theory of the strong interaction between quarks and gluons, a review the interpretations in this new key of reading necessarily arises.

The hypothesis studying a "hyperdimensional atom" explains the reason for the incomprehensible conflict "The Wave-particle duality relation" and confirms Louis de Broglie's Research on Quantum Theory hypothesized that all matter can be represented as a de Broglie wave in the manner of light.

\subsection{Heisenberg Uncertainty Principle, Why?}

The uncertainty principle is basically evincible through the electron levels in fibrational dynamic, since it is generally not possible to predict the value of a quantity with absolute certainty. In fact, any measurement test induces the parameters ( $X$ position and $P$ momentum) change in $\left(\sigma_{\bar{\omega}}\right)$ of the fibers, just as demonstrated by Heisenberg's uncertainty principle. 


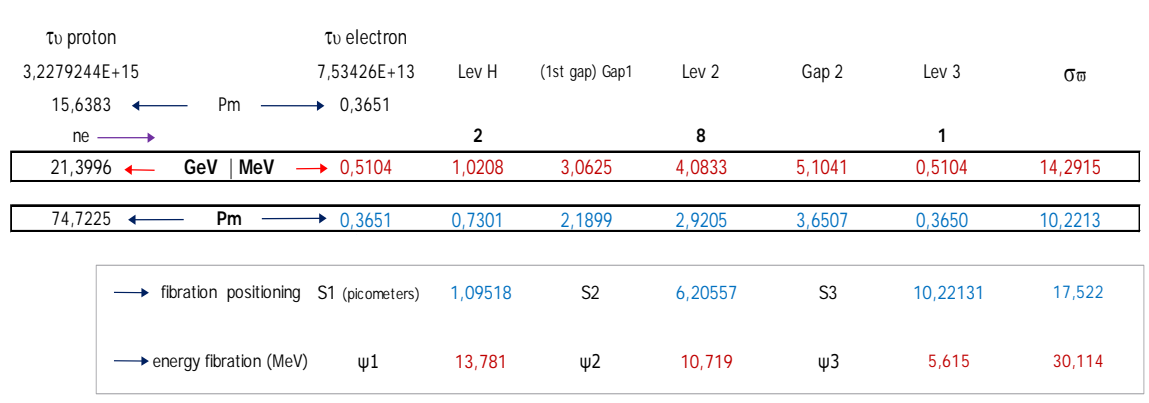

Figure 13. Preliminary development of orbital levels in terms of Hopf's fibration.

$>\Delta_{\chi} \rightarrow f \Delta S^{x n}\left(\tau_{v}\right)$

$>\Delta_{P_{X}} \rightarrow f \Delta S^{p n}\left(\sigma_{\omega}\right)$

\subsection{How Is Gravitational Force Generated and Why Is It Always Attractive?}

We have explained the concept of time curvarture as an effect of "temporal discrepancy" originating between the chronotropic mass referred to the event and the spacetime indicated in Planck time defined as precursive time.

The Equations (1) and (2) indicate that the mass and time curvature are proportional. This leads us to infer that time curvature is primarily responsible for gravitational mass.

In fact, Equation (6) confirms the thesis:

$$
g=G \frac{m_{1}}{r^{2}} \Rightarrow g=G \frac{\psi}{r \Delta \tau}
$$

- ge gravity.

- r. radius.

- G: gravitational constant.

- $\Delta \tau$ : time-space curvature.

The reason why the inertial mass and gravitational mass are related is because both forces originate from the same causal event.

The direct correlation exists between inertial and gravitational mass, so the gravitational mass value acquires an objective value defined Gravitation as a $<$ specific attraction degree $\left(A_{0}\right)>$.

$$
A_{0} \equiv \Delta_{q \tau} \equiv g
$$

Time curvature defines the value of the specific attraction capacity degree of a body $\left(A_{0}\right)$.

The two masses attract each other $g=\left(F A_{01}-F A_{02}\right)$ so the result is the difference between the two sections of time curvature (Interferential of the Fibonacci areas).

Brief note about it.

In gravitational interactions, areas $(F)$ are consistently added into quaternion matrix as described according to Hurwitz-Lipschitz, since the components are unitary quantities. 
However, in this study we limit the exposure to the gravitational mass equation or gravitational attraction force, understood as a specific attraction capacity of a body as a function of the time curvature (space deflated effect). About the interactions between bodies, that is, between gravitational masses, the theory extends the concept to the principle of interference, resulting from the mutual action of the precursive areas $\left(e^{q-}\right)$ (Figure 14).

\section{Discussion}

Compared to the first publication of the theory, we collected reviews from many colleagues who highlighted the difficulty in understanding exactly the sense of time curvature and precursive time. The difficulty lies in acquiring a new knowledge of "binary" matter including of a hyper-dimensional part. As explained, the study basically demonstrates that matter and spacetime are two constituents of a single entity. The same principle is applicable on a nuclear scale by establishing a correlation at the base of the electroweak force, between the nucleus and the electronic orbitals, so even the Higgs boson, can be considered as the minimum time discrepancy amount that can give rise to a ponderable unit of mass through the LHC.

Some colleagues asked us if the theory went towards a GUT hypothesis or if ToE, given that in the same work it is exposed to gravitational force and electroweak force. However, the fact that the publication exposes aspects of general relativistic physics, and nuclear physics in the same study, confirms the hypothesis of a Theory of ToE Unification. This made it difficult even to characterize the physics area for the publication of this study.

As a final point, we can ask ourselves whether there are any scientific clues leading to acceptance of this hypothesis.

Regarding this difficult question we are sure that the theoretical research was born from the need to identify a scientific hypothesis consistent with the theories and phenomenologies gained, to provide an interpretation of the inexplicability/conflictuality of the current physics. To this aim, we can say that until now neither evidence nor clues have emerged, that exclude it from a valid research hypothesis.

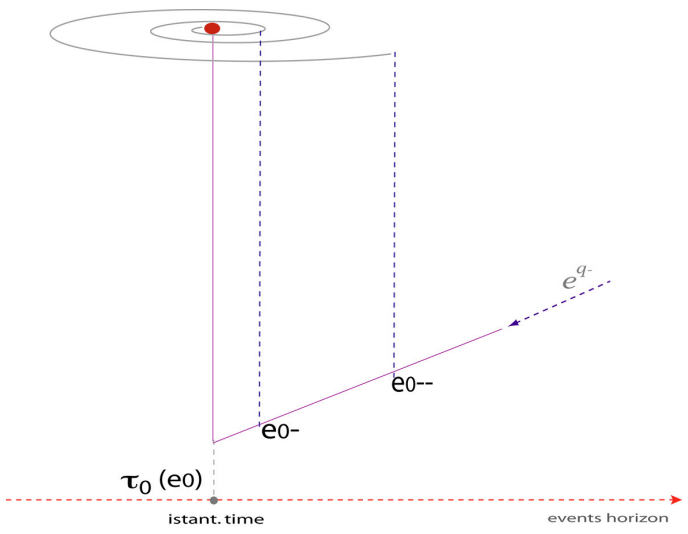

Figure 14. $\left(e_{q_{-}}\right)$: precursive time events. 


\section{Conclusions}

In this study we have made a subsequent deepening of the theoretical hypothesis of temporal curvature as the basic phenomenon of the interaction between matter and spacetime. The study summarized the electroweak interaction between the nucleus and orbital levels, applying the same equations describing spacetime and gravitation.

The study pointed out the following demonstrations:

1) CORRELATION: TIMESPACE CURVATURE $\Leftrightarrow$ NUCLEAR ENERGY

2) CORRELATION: TIMESPACE CURVATURE $\Leftrightarrow$ ELECTRON ENERGY LEVELS

3) CORRELATION: NUCLEAR MASS $\Leftrightarrow$ NUCLEAR ELECTRON ORBITAL ENERGY LEVELS

4) CORRELATION: $\Delta \tau \rightarrow \Gamma_{p}$ Exothermic $\leftrightarrow$ endothermic $Q$ Value

Mathematical analyses have been demonstrated with Equations: (1) (3)-(6).

From Equation (1) we calculate:

1) Time curvature (Planck time)

2) Precursive time (joule/second) inertial mass

3) Timespacedeflation $(\sigma)$ (second $\left.{ }^{*} c^{2}\right)$ gravitational mass

4) Nuclear Electronic orbital (Planck time ${ }^{\star} c^{2}$ ) - Electroweak interaction.

From Equation (3) we calculate:

Mass-matter or "hypermatter": calculation of the mass as a function of the induced space-time curvature.

Equation (3) modifies the concept of mass intended as a scalar quantity, replaces the concept of quantitative aggregate as a derivative of a function composite. The symbol $(\psi)$ indicates the complex mass.

The equation introduces two new relationships:

1) Quantum specific heat capacity (QSHC): Planck temperature as a function of time curvature.

2) QCS $\left(\pi^{F}\right)$ coefficient

The processing of the graphical representation models has characterized the QCS $\left(\pi^{F}\right)$ coefficient, allowing to reproduce the centripetal dynamics moment of the twisting effect of spacetime.

From Equation (5) we calculate: energy equation: $E=m c^{2} \equiv \psi c^{3}$

The ability to calculate the energy ( $\left.E=m c^{2}\right)$ through the hypermatter calculation equation $(\psi)$, confirms consistency.

The study has been carried out according to a pragmatic theoretical development:

$<$ Idea $\rightarrow$ hypothesis $\rightarrow$ thesis $\rightarrow$ theory $>$

The following stages were developed: conceptual analysis; topological and mathematical conceptual connections; analysis of phenomenological coherence with classical theories.

Key concepts elaborated: 
- Timespace correlative manifold variety

- Quaternion algebra; hyperbolic geometry - hypersphere - Minkowski spacetime

- Quadrant Complex depiction (complex time + complex space)

- Quantum compensation spacetime $\left(\pi^{r}\right) ;(F)$ Fibonacci coefficient

- Hopf fibration mathematical structure The study was subject to "Theoretical Stress Test":

- Inconsistencies

- Theoretical inconsistencies

- Phenomenological inconsistencies

- Calculation inconsistencies

\section{Theoretical Definitions}

In the study unpublished definitions were suggested to make exposure understandable. However, the study proposes them as investigational indicators, suggested to the scientific community for all changes that the research will consider necessary:

Time curvature: It is understood as the discrepancy timethat occurs between inertial frame of reference and spacetime. The theoretical research that has been carried out has shown that the spacetime curvature is a phenomenon resulting from the complex interaction between the three exclusive ingredients that make up our universe: matter-space-time. The notion makes it possible to combine and unify quantum physics with classical and relativistic theories.

Precursive time: defines the value of the time curvature in quantities of Planck time or $(-\mathrm{sec})$ of. It is the variable that quantifies the temporal curvature that the mass induces on spacetime. It can be considered as the physical mediator between matter and spacetime.

Space deflation - Time deflection: In the twist representation, the contraction of space (deflation) is related to a reduction of time (deflection). In summary, it takes less time to travel less space.

Chronotope: Indicates the spatial position of an event at a specific instant time. The chronotope establishes a condition of exclusivity, as there can be no overlap of events having the same space and time coordinates

Mass-matter. The equation with symbol $(\Psi)$, enshrines a concept of complex mass.

Timespace manifold: The topological variety of relationships between Minkowski space and the projection of the complex time variable. The variety was developed to represent in a comprehensible form the points of conjunction between the real parameters of the event and its imaginary projection. The connection is hypothesized according to the principle of entanglement correlation.

Timespace Quadrant. Representation chart of all real and complex components of the event at the time instant.

Q. Heat capacity: Planck temperature related to time curvature. It is a parameter for the calculation of mass-matter. It expresses a thermal characteristic of 
matter $(m i>0)$ as a function of spacetime.

Q.c.s. Quantum compensation spacetime $\left(\pi^{F}\right)$ : coefficient for the calculation of the mass-matter. It determines the angular momentum of spacetime.

$\left(A_{0}\right)$ specific attraction degree: The study shows that inertial and gravitational mass derive from the same cause described as time curvature. It follows that a body's gravitational attraction force can be simply resolvedas a specific attraction degree.

\section{Acknowledgements}

I wish to thank all my trusted and intangible collaborators for all the support that they have poured into me during these years of study, starting with: Hermann Minkowski who despite his extraordinary synthesis of spacetime in the science of a thousand inexplicables is still somewhat unrecognized. To Albert who is always willing to whisper advice to me, to David Bohm whom I consider my putative father. I thank all the other collaborators for what they have given me their knowledge: Max Planck, W. R. Hamilton, Ludwig Boltzmann, J. H. Poincaré, J. Krishnamurti, Max Born. Last but not least to Giordano Bruno who feed the flame of my knowledge.

\section{License}

The publication is licensed under Academic Free License (“AFL") v. 3.0 a Creative Commons Attribution 4.0 International License.

\section{Conflicts of Interest}

The authors declare no conflicts of interest regarding the publication of this paper.

\section{References}

[1] Minkowski, H. and Born, M. (1910) EineAbleitung der Grundgleichungenfür die elektromagnetischen Vorgänge in bewegten Körpern. Mathematische Annalen, 68, 526-551. https://doi.org/10.1007/BF01455872

[2] Minkowski, H. (1890) H. Poincaré, Sur le problème des trois corps et les équations de la dynamique. Jahrbuchüber die Fortschritte der Mathematik, 22, 907-914.

[3] Minkowski, H. (1896) Geometrie der Zahlen. Teubner, Leipzig.

[4] Minkowski, H. (1907) Kapillarität. See Encyklopädie der mathematischen Wissenschaftenmit Einschlussihrer Anwendungen V, Physik, Volume 1, Sommerfeld, 558-613. https://doi.org/10.1007/978-3-663-16016-8 10

[5] Minkowski, H. (1908) Die Grundgleichungenfür die electromagnetischen Vorgänge in bewegten Körpern. Nachrichten von der Königlichen Gesellschaft der Wissenschaftenzu Göttingen, 53-111.

[6] Minkowski, H. (1909) Raum und Zeit. Jahresbericht der deutschen Mathematiker-Vereinigung, 18, 75-88.

[7] Minkowski, H. (1915) Das Relativitätsprinzip. Jahresbericht der deutschen Mathematiker-Vereinigung, 24, 372-382. https://doi.org/10.1002/andp.19153521505 
[8] G. B. M. (1912) Bibliography of Non-Euclidean Geometry, including the Theory of Parallels, the Foundations of Geometry, and Space of N Dimensions. Nature, 89, 266. https://doi.org/10.1038/089266a0

[9] Donaldson, S.K. (1983) An Application of Gauge Theory to Four-Dimensional Topology. Journal of Differential Geometry, 18, 279-315. https://doi.org/10.4310/jdg/1214437665

[10] Freedman, M.H. (1982) The Topology of Four-Dimensional Manifolds. Journal of Differential Geometry, 17, 357-453. https://doi.org/10.4310/jdg/1214437136

[11] Solov'ev, Y.P. (1991) The Topology of Four-Dimensional Manifolds. Russian Mathematical Surveys, 46, 167. https://doi.org/10.1070/RM1991v046n02ABEH002744

[12] Gille, P. and Szamuely, T. (2006) Central Simple Algebras and Galois Cohomology. Cambridge Studies in Advanced Mathematics, Vol. 101, Cambridge University Press, Cambridge.

[13] Lorentz, H.A., Einstein, A., Minkowski, H. and Weyl, H. (1952) The Principle of Relativity: A Collection of Original Memoirs on the Special and General Theory of Relativity. Dover Publications, New York.

[14] Ehrenfest, P. (1920) How Do the Fundamental Laws of Physics Make Manifest That Space Has 3 Dimensions? Annalen der Physik, 61, 440. https://doi.org/10.1002/andp.19203660503

[15] Cayley, A. (1845) On Certain Results Relating to Quaternions. Philosophical Magazine, 26, 141-145. https://doi.org/10.1080/14786444508562684

[16] Hurwitz, A. (1919) Vorlesungenüber die Zahlentheorie der Quaternionen. Springer, Berlin. https://doi.org/10.1007/978-3-642-47536-8

[17] Burri, R. (2016) Vacuum-Matter Interaction through Hyper-Dimensional Time-Space Shifting. Journal of High Energy Physics, Gravitation and Cosmology, 2, 432-446. https://doi.org/10.4236/jhepgc.2016.23037

[18] Glaister, P. (1995) Fibonacci Power Series. The Mathematical Gazette, 79, 521-525. https://doi.org/10.2307/3618079

[19] Hameroff, S. and Penrose, R. (2014) Consciousness in the Universe: A Review of the "ORCH OR" Theory. Physics of Life Reviews, 11, 39-78. https://doi.org/10.1016/j.plrev.2013.11.013

[20] Bugeaud, Y., Mignotte, M. and Siksek, S. (2006) Classical and Modular Approaches to Exponential Diophantine Equations. Annals of Mathematics, 163, 969-1018. https://doi.org/10.4007/annals.2006.163.969

[21] Prusinkiewicz, P. and Hanan, J. (1989) Lindenmayer Systems, Fractals, and Plants (Lecture Notes in Biomathematics). Springer-Verlag, Berlin. https://doi.org/10.1007/978-1-4757-1428-9

[22] Battelli, L., Bianchi-Streit, M. and Giacomelli, G. (1993) Particle Physics with Bubble Chamber Photographs (Report). CERN, 3-4.

[23] Hopf, H. (1931) Über die Abbildungen der dreidimensionalen Sphäre auf die Kugelfläche. Mathematische Annalen, 104, 637-665. https://doi.org/10.1007/BF01457962

[24] Mosseri, R. and Dandoloff, R. (2001) Geometry of Entangled States, Bloch Spheres and Hopf Fibrations. Journal of Physics A: Mathematical and Theoretical, 34, 10243-10252. https://doi.org/10.1088/0305-4470/34/47/324

[25] Zabrodsky, A. (1976) Hopf Space. Hebrew University, Jerusalem, North-Holland Publishing, Amsterdam. 